\title{
A decade of dark matter searches with ground-based Cherenkov telescopes ${ }^{\text {th }}$
}

\author{
Michele Doro \\ University and INFN Padova, via Marzolo 8, 35131 Padova (Italy) \\ Department of Physics and CERES, Campus Universitat Autónoma Barcelona, 08135 Bellaterra (Spain)
}

\begin{abstract}
In the general scenario of Weakly Interacting Massive Particles (WIMP), dark matter (DM) can be observed via astrophysical gamma-rays because photons are produced in various DM annihilation or decay processes, either as broad-band or line emission, or because of the secondary processes of charged particles in the final stages of the annihilations or the decays. The energy range of the former processes is accessible by current ground-based Imaging Atmospheric Cherenkov telescopes (IACTs, like H.E.S.S., MAGIC and VERITAS). The strengths of this technique are: a) the expected DM gamma-ray spectra show peculiar features like bumps, spikes and cutoff that make them clearly distinguishable from the smoother astrophysical spectra, b) the expected DM spectrum is universal and therefore by observing two or more DM targets with the same spectrum, a clear identification (besides 'detection) of DM would be enabled. The role of IACTs may gain more importance in the future as the results from the LHC may hint to a DM particle with mass at the $\mathrm{TeV}$ or above, where the IACTs sensitivity is unsurpassed by other experiments. In this 'contribution, a review of the search for DM with the current generation of IACT will be presented.
\end{abstract}

Keywords: IACT, gamma-ray astronomy, dark matter, H.E.S.S., MAGIC, VERITAS, CTA

\section{Introduction}

One of the most interesting and compelling observations that ground-based Cherenkov telescopes operating in the veryhigh-energy gamma-ray band can perform is that looking at targets in the sky where a large concentration of dark matter (DM) is expected. There are several reasons in support of this. First of all, DM is indeed expected in the sky. $80 \%$ of the total matter 'content of the Universe is constituted by one or more new types of particles. The DM has shaped the formation of the first stars and galaxies, so thoroughly that the concordance cosmological model is called $\Lambda$ CDM where CDM stands for Cold DM. We also know that there are places in the sky where DM is expected to be particularly concentrated. We don't know the DM nature 'and if it could finally be detected via primary or secondary radiation associated with its annihilation or decay, but there are 'several models that predict such signatures and it is therefore worth "sailing" our telescopes to these promised lands, despite no "Earth!" signal has arrived so far. Secondly, the gamma-ray -band is a very privileged one, for several reasons: a) gammarays are neutral and trace back to the point of origin, where we expect DM, b) the gamma-ray spectrum emerging from DM interactions (either annihilations or decays) is universal. All DM targets are expected to show exactly the same gamma-ray spectrum. The observation of multiple spectra from different targets would therefore constitute an excellent result, c) gamma-ray spectra from DM annihilations or decay typically show several characteristic features, naturally depending on the specific dark

The original title of the invited talk was "Dark matter searches with Cherenkov Telescopes". This version has some typo corrections with respect to the published version, as well as an updated Fig. 2. matter type, but in general classifiable in sharp cutoff, bumps, or even line emissions. This makes the DM spectra hardly confusable with typical astrophysical spectra. Third, the recent experimental results of the LHC experiments: the quite large Higgs boson mass and the non-evidence for New Physics beyond the Standard Model are possibly hinting to DM particle being more massive than expected, about the $\mathrm{TeV}$ or above [1]. The $\mathrm{TeV}$ region is where ground-based telescopes have highest sensitivity.

And indeed this is what was done in the last decade, specially with the H.E.S.S., MAGIC and VERITAS experiments. These very successful experiments, all together, invested quite a large fraction of their observation times in the last years to cover the targets where DM was expected. In this contribution, we will try to review these observations. For additional details on gamma-ray signals from dark matter, we refer the reader to Ref. [2].

\section{Ground-based imaging Cherenkov telescopes}

Gamma rays produced in Space cannot cross the Earth atmosphere. After few radiation lengths, they interact with the electrostatic field of the atmospheric atoms and convert into an electron-positron pair. However, these leptons are extremely energetic (they share the energy of the primary gamma ray), and therefore are able to initiate an electromagnetic showers thanks to subsequent emission of bremsstrahlung gamma rays which in turn pair produce again. The shower is few $\mathrm{km}$ long and few hundreds of meter large. It has a maximum at about 10$12 \mathrm{~km}$ above the ground (at the $\mathrm{GeV}-\mathrm{TeV}$ ) and dies out when the lepton energy reaches the threshold for ionization, which in 
air is $83 \mathrm{MeV}$. Even if the shower particles are lost in the atmosphere, the fact that most of the time the leptons were travelling faster than the speed of light in the atmosphere, allowed for the radiation of Cherenkov light from the atmospheric medium swept up by the shower. This light has a continuous spectrum, peaked at about $300 \mathrm{~nm}$ after the ozone absorption, and it is a flash of light of the duration of few ns, expanding within a cone of aperture of about 1 deg radius, which at the ground illuminates an area of about $100 \mathrm{~m}$ radius. For this reason, if one puts a telescope in this Cherenkov light pool, the "effective" area of the telescope is not simply the geometrical area of the dish, but almost the entire Cherenkov pool, which sums up to about $10^{5} \mathrm{~m}^{2}$. One could compare this number with the typical areas of satellite-borne gamma-ray detectors, of about $1-2 \mathrm{~m}^{2}$, hardly expandable in the near future. The drawback is that gamma-ray showers at few $\mathrm{GeV}$ produce too little Cherenkov photons to be detected, and the technique is fully sensitive at the $\mathrm{TeV}$ scale and above.

Ground-based Cherenkov telescopes are also called Imaging Atmospheric Cherenkov Telescopes (IACTs) because they "image" the shower, in the sense that if a multi-pixel camera is placed at the telescope focal plane, the image of a typical electromagnetic shower is an oblate ellipse pointing to the center of the camera (corresponding to an inclined section of the Cherenkov light cone). Through the careful analysis of shower primary, secondary and sometimes tertiary moments, and in some cases its time evolution, one can reconstruct the energy and direction of the primary gamma ray. The main source of background is constituted by the showers generated by hadronic cosmic rays. These are mixed sub-hadronic and sub-electromagnetic showers initiated by primary cosmic rays in the top atmosphere like protons, helium, and heavier nuclei. They are by far more abundant than gamma rays, which are seen roughly every 10,000 hadrons. Therefore, the technique relies on a first telescope topological trigger system that rejects about $99 \%$ of the hadrons, and later on a gamma/hadron separation at the analysis level. The technique was pioneered by the Whipple observatory, that after tens of years of hunting, finally detected the Crab Nebula in 1989, and it is now superseded by the H.E.S.S., MAGIC and VERITAS arrays.

Table 1 collects some information regarding the major IACT experiments. We mention also that the Whipple telescope terminated operation this year.

\begin{tabular}{l|ccc}
\hline IACT & Year & Nr. tels \& diameter & Location \\
\hline Whipple & 1968 & $1 \times 12 \mathrm{~m}$ & Arizona, USA \\
H.E.S.S. & 2003 & $4 \times 12 \mathrm{~m}+1 \times 28 \mathrm{~m}$ & Gambserg, Namibia \\
MAGIC & 2004 & $2 \times 17 \mathrm{~m}$ & La Palma, Spain \\
VERITAS & 2007 & $4 \times 12 \mathrm{~m}$ & Arizona, USA \\
\hline
\end{tabular}

Table 1: Current major operating ground-based Cherenkov telescopes. Given are the starting year, the array multiplicity and dish diameter in the latest configuration, and the location.

\section{Gamma-ray signatures from dark matter}

There is no space in this review to account for all the particles that are valid candidate for DM. We refer to [3] for a re- cent review. In this context, we concentrated more on a Weakly Interacting Massive Particle (WIMP) scenario, which foresees a particle at the GeV-TeV scale, whose annihilation or decay products are found in the Standard Model (SM) particle zoo. There are valid scenarios of decaying DM, however, they are not furtherly treated here.

Now, if DM is coupled to the SM with some interactions, in the final products of annihilations or decays one can find either leptons or hadrons or gauge bosons, often the heaviest one because of the scale of the DM mass, which is at the GeV-TeV. It is therefore expected naively to find also gamma-rays in the final products. More precisely, the gamma-ray emission can be originated as follows (see Fig. 1): $a$ ) from neutral pion decays after hadronization of quarks. This gives origin to a broadband spectrum terminating with a cutoff at the DM mass; $b$ ) from final state radiation of leptons. This also gives origin to a broadband spectrum and a cutoff but with harder photons; $c$ ) gamma rays from internal bremsstrahlung, when the annihilation is to sfermions and the annihilation is in the t-channel, which gives rise to a pronounced bump of gamma rays toward the mass cutoff [2]; $d$ ) from line-processes ending in $\gamma X$, where $X$ could be $\gamma, Z_{0}, h$. These are loop processes, whose intensity strongly depends on the specific DM realization, which give rise to line emissions that constitute smoking guns detection for DM, because an astrophysical explanation of these lines would be extremely challenging. There are other gamma-ray emission processes, following other particle models, which are not described here.

All in all, IACTs observe photons. Therefore, every process is valuable as long as it provides enough photons to detect. However, the more features the spectrum exhibits, the easier is the consequent DM identification. In this sense, not only the total flux is relevant. Generally, the DM annihilation flux in gamma rays is expressed as:

$$
\frac{d \Phi}{d E}(E ; \Delta \Omega)=\frac{B_{F}}{4 \pi} \frac{\left\langle\sigma_{a n n} v\right\rangle}{2 m_{\chi}^{2}} \frac{d N_{\gamma}}{d E} \int_{\Delta \Omega} \int_{l o s} d \theta d s \rho^{2}(\theta, s)
$$

where $\left\langle\sigma_{\mathrm{ann}} v\right\rangle$ is the averaged annihilation cross-section times the velocity, $m_{\chi}$ the DM particle mass, $d N_{\gamma} / d E$ is the total number of photons produced during one annihilation event, and their product is conventionally called the particle physics factor. The second term of the equation is called the astrophysical factor or J-factor and it is computed as the line of sight $s$ integral of the square of the DM density, and over a certain solid angle $\Delta \Omega=2 \pi(1-\cos (\theta))$ under which the source is observed. Finally, $B_{F}$ is the so-called intrinsic boost factor, and it is a measure of the uncertainty in either the particle physics or the astrophysics terms for unaccounted intrinsic contributions to the flux. In case of of decaying DM, in the particle physics factor of Eq. 1, the term $\left\langle\sigma_{\mathrm{ann}} v\right\rangle / m_{\chi}^{2}$ is replaced by $\Gamma_{\mathrm{dec}} / m_{\chi}$ where $\Gamma_{\mathrm{dec}}$ is the inverse of the particle lifetime, and the dependence on the DM density $\rho$ is linear and not quadratic.

\section{Overview of current experimental results}

The search for DM with Cherenkov telescopes has started together with the full establishment of the IACT technique, and 


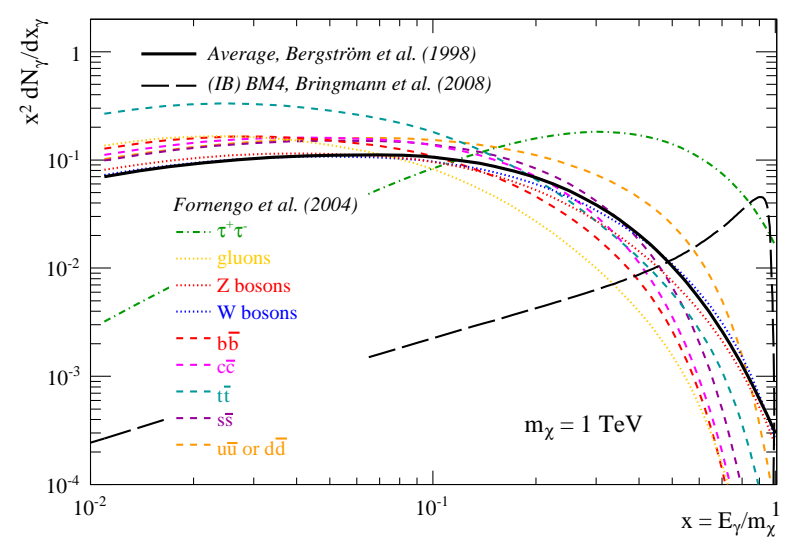

Figure 1: Differential spectra (multiplied by $x^{2}$ ) of gamma-rays from several neutralino annihilation products. Taken from [4, Fig. 1].

namely with the H.E.S.S., MAGIC, VERITAS and Whipple experiments. Table 2 lists the various targets and searches performed, organized by target class, and reporting the year and duration of the observation (in hours), and the reference publication (or proceedings). I apologize for possible missing entries in this table.

As a general trend, the searches started with the target classes that 10 years ago were supposed to be the best candidate: the galactic center, galaxy clusters and dwarf satellite galaxies. More recently, the attention has been also focused on different kinds of searches, like those at intermediate mass black holes or DM subhalos and at signatures of line-emission. Finally, - specially raised by the all-electrons and positron ratio anomalies observed in the past 5 years, discussed later- the DM searches have also been extended to signatures from cosmic ray leptons rather than gamma-rays, an observation which is also possible with IACTs. In the following, an overview class by class is given.

\subsection{Observation of dwarf satellite galaxies}

The Dwarf Satellite Galaxies (DSGs, often called dwarf spheroidal galaxy or dSphs) are rather small (order $10^{7} \mathrm{M}_{\odot}[31]$ ) galaxies, gravitationally bound to the Milky Way, located in the Milky Way dark matter halo, at distances below $250 \mathrm{kpc}$. Cosmological N-body simulations $[32,33]$ predict that, besides the main "smooth" DM halo, a wealth of substructures should be present down to scales of $10^{-6}-10^{-12} \mathrm{M}_{\odot}$. Onto these overdensities, baryons could have accreted until starting star and galaxy formation, however, the impact of baryon physics at these targets is probably smaller compared to Milky Way sized objects. Because of the relatively low star content and gravitational pull, most of the DSG went inactive long time ago, with no major stellar activities found. Their total number is an issue, with discrepancies between observation data and simulations (the socalled "missing satellite problem" [34]), and so far only about twenty were discovered. Also their relation with visible galaxies is subject of debate, because the predictions foresee very large DSG that are not in fact observed, the so-called "too big to fail" problem [35]. They are standardly classified in "classical" and "ultra-faint". The latters usually refer to those discovered

\begin{tabular}{|c|c|c|c|c|}
\hline Target & Year & Time & Experiment & Ref. \\
\hline \multicolumn{5}{|c|}{ Globular Clusters } \\
\hline \multirow[t]{2}{*}{ M15 } & 2002 & 0.2 & Whipple & {$[5]$} \\
\hline & $2006-2007$ & 15.2 & H.E.S.S. & {$[6]$} \\
\hline M33 & $2002-2004$ & 7.9 & Whipple & [5] \\
\hline M32 & 2004 & 6.9 & Whipple & {$[5]$} \\
\hline NGC 6388 & $2008-2009$ & 27.2 & H.E.S.S. & {$[6]$} \\
\hline \multicolumn{5}{|c|}{ Dwarf Satellite Galaxies } \\
\hline \multirow[t]{3}{*}{ Draco } & 2003 & 7.4 & Whipple & {$[5]$} \\
\hline & 2007 & 7.8 & MAGIC & [7] \\
\hline & 2007 & 18.4 & VERITAS & {$[8]$} \\
\hline \multirow[t]{2}{*}{ Ursa Minor } & 2003 & 7.9 & Whipple & {$[5]$} \\
\hline & 2007 & 18.9 & VERITAS & [8] \\
\hline Sagittarius & 2006 & 11 & H.E.S.S. & [9] \\
\hline Canis Major & 2006 & 9.6 & H.E.S.S. & [10] \\
\hline \multirow[t]{2}{*}{ Willman 1} & $2007-2008$ & 13.7 & VERITAS & {$[8]$} \\
\hline & 2008 & 15.5 & MAGIC & [11] \\
\hline Sculptor & 2008 & 11.8 & H.E.S.S. & [12] \\
\hline Carina & $2008-2009$ & 14.8 & H.E.S.S. & {$[12]$} \\
\hline \multirow[t]{3}{*}{ Segue 1} & $2008-2009$ & 29.4 & MAGIC & [13] \\
\hline & $2010-2011$ & 48 & VERITAS & [14] \\
\hline & $2010-2013$ & 158 & MAGIC & [15] \\
\hline Boötes & 2009 & 14.3 & VERITAS & [8] \\
\hline \multicolumn{5}{|c|}{ Galaxy Clusters } \\
\hline Abell 2029 & $2003-2004$ & 6 & Whipple & [16] \\
\hline \multirow[t]{2}{*}{ Perseus } & $2004-2005$ & 13.5 & Whipple & [16] \\
\hline & 2008 & 24.4 & MAGIC & [17] \\
\hline Fornax & 2005 & 14.5 & H.E.S.S. & [18] \\
\hline Coma & 2008 & 18.6 & VERITAS & [19] \\
\hline \multicolumn{5}{|c|}{ The Milky Way central region } \\
\hline MW Center & 2004 & 48.7 & H.E.S.S. & {$[20]$} \\
\hline MW Center Halo & $2004-2008$ & 112 & H.E.S.S. & {$[21]$} \\
\hline \multicolumn{5}{|c|}{ Other searches } \\
\hline \multirow[t]{2}{*}{$\overline{\mathrm{IMBH}}$} & $2004-2007$ & 400 & H.E.S.S. & {$[22]$} \\
\hline & $2006-2007$ & 25 & MAGIC & [23] \\
\hline \multirow[t]{2}{*}{ Lines } & $2004-2008$ & 112 & H.E.S.S. & {$[24]$} \\
\hline & $2010-2013$ & 158 & MAGIC & {$[15]$} \\
\hline \multirow[t]{2}{*}{ UFOs } & - & - & MAGIC & {$[25]$} \\
\hline & - & - & VERITAS & [26] \\
\hline \multirow[t]{2}{*}{ All-electron } & $2004-2007$ & 239 & H.E.S.S. & {$[27,28]$} \\
\hline & $2009-2010$ & 14 & MAGIC & [29] \\
\hline Moon-shadow & - & - & MAGIC & {$[30]$} \\
\hline
\end{tabular}

Table 2: Compound of observational targets for indirect dark matter searches with gamma rays from the Whipple, H.E.S.S., MAGIC and VERITAS experiments. In the second and third columns, the year and duration of observations (in hours) are given. In the last column, link to papers (or proceedings if papers were not available) are given.

after the Sloan Digital Sky Survey (SDSS) spectrometric experiment, but shadows a more subtle difference: ultra-faint DSGs have more compact sizes, less stars, and are more DM dominated than the classical, with mass-to-light ratio sometimes exceeding $1000 M_{\odot} / L_{\odot}$. Nowadays we know the existence about two dozens DSGs in the MW halo. Among the classical DSGs we have Sculptor, Fornax, Leo I and Leo II, Draco, Ursa Minor, Carina, Sextans while among the ultra-faint DSGs we find: Willman 1, Ursa Major I and II, Hercules, Leo I, II, IV, Canes Venatici I and II, Coma Berenices, Segue I and Bootes I and others. DSGs are extremely interesting to observe, for the rea- 
son that the dynamic of the object is strongly steered by DM, and the baryons play a secondary role. This means that not only the gamma-ray contribution from possible standard astrophysical sources (e.g. supernova activity) is low, but also that the determination of the DM content itself from the stellar velocity distribution is relatively precisely determinable. The Jfactor is computed in DSGs via the Jeans hydrostatic equilibrium equation from the measured stellar position and velocities of the DSG member star. To solve the equation one needs to input a model for the star phase-space and another one for the DM density profile, whose normalization is left as a free parameter. All the details of these calculations are reviewed in Ref. [36]. As an example, for the Segue 1 DSG, considered as one of the most dark-matter dominated objects, Ref. [37] reports uncertainties in the J-factor of one order of magnitude: $\log _{10}\left(J_{\text {Segue }}\right)=19.0 \pm 0.6 \mathrm{GeV}^{2} \mathrm{~cm}^{-5}$. On the other hand Segue 1 has only 71 confirmed member stars, a factor ten less than the classical DSGs like Draco, which is probably the best constrained classical DSGs with a thousand of identified member stars.

Since 2004, these targets have extensively studied by IACTs $[5,7,8,9,10,11,12,13,14,15]$. Sagittarius, present in Tab. 2 is not included in the list above of classical DSGs because of its peculiar nature, with extremely extended tails, which makes very uncertain the estimation of its DM content. However, we mention that this conclusion is under debate, and H.E.S.S. has recently shown results with a total observation time on this target larger than $90 \mathrm{~h} \mathrm{[38].} \mathrm{The} \mathrm{table} \mathrm{shows} \mathrm{that} \mathrm{during} \mathrm{the} \mathrm{first}$ years, different targets have been observed for limited amount of hours, typically below 20 hours, while more recently, and specially for the DSG Segue 1, the amount of hours dedicated to a single source has extremely increased. A first motivation is that back in 2004, the prospects of detection were more optimistic [39]. At that times, only the classical DSGs were known, and it was obvious to try to observe one or more of these objects, and among these the Draco DSG which was considered one of the most promising. After the advent of the SDSS experiment, and the continuous discovery of new ultra-faint DSGs, the attention was turned to these new targets, for whom the predictions were extremely exciting. Obviously, the exclusion limits obtained with the first publications about the classical DSGs quite improved in the latest publications, with extended observation times, and often improved analysis techniques. It is worth mentioning the application of a dedicated full likelihood method in the deep Segue 1 observation with the MAGIC-stereo system [40], where the authors improved the sensitivity of the telescopes by up to a factor of 2 by fully taking into account the specific spectral features of typical DM spectra, as well as the telescope energy resolution. This method, applied to DM searches, has so far provided the most constraining results for indirect DM searches at DSGs with IACTs, see also Fig. 2. Another specific method based on Event Weighting is developed by VERITAS experiment [41], that improves the sensitivity from a joint analysis of individual targets into a single limit and utilizing more of the individual event information.

It is safe to say that the exclusion limits obtained with DSGs are possibly the most robust for indirect DM searches with IACTs with upper limits reaching cross-section values of the order of $10^{-24} \mathrm{~cm}^{3} \mathrm{~s}^{-1}$.

\subsection{Observation of globular clusters}

The globular clusters share some properties with DSGs, but in general are less bright and less massive. On the other hand, the stellar population is normally different, with globular clusters having more homogeneous stellar content in terms of star classification. The debate on whether they reside in a DM halo is still open. From the cosmological point of view, there is no conflict in telling that they were formed in DM overdensities, as the DSGs, however, there are no strong observational evidence for the presence of a DM core. A possibility is also that they possessed DM cores early disrupted by tidal forces.

The M15, M32 and M33 globular clusters were observed from 2002 to 2004 by Whipple [5] and later on M15 was reobserved together with NGC6388 by H.E.S.S. in 2006-2009 [6]. The best exclusion curves for annihilating DM come from the H.E.S.S. results, and are the order of $10^{-24}-10^{-25} \mathrm{~cm}^{3} \mathrm{~s}^{-1}$, however they rely on strong assumptions of the dominance on $\mathrm{DM}$ in these objects.

\subsection{Observation of subhalos}

We have already observed that standard N-body simulations and cosmological theories predict the existence of small DM overdensities at all scales within the main smooth halo. Some of these "subhalos" could have been too small to have attracted enough baryonic matter to initiate star formation and would therefore be invisible to past and present astronomical observations at all wavelengths. However, gamma rays could be expected from those objects due to annihilations or decays of DM [42]. A possible way to observe them is with gammaray all-sky monitoring programs, like those conducted by the EGRET [43] and Fermi-LAT satellite [44]. A population with no counterparts at other wavelengths and similar spectra could indeed be associated to subhalos.

Following this idea, the MAGIC and VERITAS collaboration investigated among the unidentified Fermi objects (also called UFOs), those with no obvious counterparts, satisfying some criteria in the stability of the spectrum, and spectral hardness that could be explained as subhalos [45]. Few targets were observed by both experiments, however without any hint of detection, and results are under publications $[26,25]$.

\subsection{Observation of intermediate mass black holes}

There are theories that predict the existence of black holes of mass comprised between $10^{2}-10^{6} \mathrm{M}_{\odot}$, sometimes referred to as Intermediate Mass Black Holes (IMBHs). Many of them could reside in the Milky Way halo. They could form as remnants of collapse of Population III stars (scenario A, $10^{2} \mathrm{M}_{\odot}$ ). The average number of IMBHs in the MW halo was estimated by numerical simulation to be on the order of about a thousand. In a second case (scenario $\mathrm{B}, 10^{6} \mathrm{M}_{\odot}$ ), IMBHs originate from massive objects formed directly during the collapse of primordial gas in early-forming halos. The total number of IMBHs 
in this scenario would be about a hundred. As a result the increased gravitational potential due to infalling baryons on a central accreting system, the DM could have readjusted and shrunk, giving rise to the formation of what are called "mini-spikes". On the other hand, mini-spikes are rapidly disrupted as result of dynamical processes like black hole formation or merging events. The interesting thing about these objects, is that, starting from a typical Navarro, Frenk, White (NFW [46]) distribution for the DM, the adiabatic growth of the spike leads to a final DM density profile even cuspier than the NFW, with central slope of index $-7 / 3$. For scenario B, which foresees more luminous objects than scenario $\mathrm{A}$, the corresponding gammaray luminosity would be of the order of the gamma-ray luminosity of the entire Milky Way halo, which made IMBHs very interesting targets for DM searches [47].

As for the subhalo case described above, it was plausible that some IMBHs could have already been observed during all sky gamma-ray monitoring programs. After a selection of the EGRET better candidates among the unidentified sources, MAGIC observed the brightest unidentified EGRET source in 2006 for $25 \mathrm{~h}$ without detection [23]. However, the source was later on associated with a bright pulsar using Fermi-LAT data. Using $400 \mathrm{~h}$ of data collected from 2004 to 2007 in the region between -30 and +60 degrees in Galactic longitude, and between -3 and +3 degrees in Galactic latitude, H.E.S.S. [22] could exclude scenario B at a $90 \%$ confidence level for dark matter particles with velocity-weighted annihilation cross-section $\langle\sigma v\rangle$ above $10^{-28} \mathrm{~cm}^{3} \mathrm{~s}^{-1}$ and mass between $800 \mathrm{GeV}$ and 10 $\mathrm{TeV}$.

\subsection{Observation of galaxy clusters}

Within the standard $\Lambda \mathrm{CDM}$ scenario, galaxy clusters, with masses around $10^{14}-10^{15} \mathrm{M}_{\odot}$, are the largest gravitationally bound objects and the most recent structures to form [48]. They are complex objects, relevant for both cosmological and astrophysical studies, and for what concerns DM searches [see e.g. 49, and references therein]. DM, in fact, is supposed to be the dominant component of the cluster mass budget, accounting for up to $80 \%$ of its mass (the other components are the galaxies and the gas of the intra-cluster medium (ICM)). The effect of substructures contribution to the total DM annihilation flux discussed above is especially effective for galaxy clusters, where the intrinsic flux "boost" from subhalos can be of order $100-1000$, in particular compared to the case of DSGs, discussed previously, where the subhalos boost should contribute only marginally. Despite the fact that, due to their vicinity, DSGs are usually considered as the best sources for DM indirect detection, thanks to the subhalos boost, some authors claim that galaxy clusters have prospects of DM detection better or at least as good as those of DSGs [50, 49, 51]. On the other hand, in galaxy clusters, emission in the gamma-ray range is not only expected by DM annihilation. Clusters may host an Active Galactic Nucleus (AGN) and radio galaxies [52, 53]. Moreover gamma-rays are expected to be produced also from the interaction of cosmic rays (CRs) with the ICM. Such a contribution is usually found to be larger than the one predicted from DM annihilation. It thus represents an unavoidable source of background for DM searches in galaxy clusters. On the other hand, the different morphologies of DM (extended), cosmic-ray (compact) and the individual galaxies (point-like) could be used as a discriminator for the different components, as well as the obvious differences in the expected gamma-ray spectra from the various sources [54].

To date, the deepest exposures are performed with the MAGIC stereoscopic system of the Perseus cluster [55] and through the observation of the Coma cluster with VERITAS [19]. For DM searches, probably the strongest constraints come from the observation of the Fornax galaxy clusters, expected to be the most DM dominated one [18]. H.E.S.S., MAGIC and VERITAS have also made other campaigns on galaxy clusters, reporting detection of individual galaxies in the cluster, but only upper limits on any CR and DM associated emission [17, 56, 57, 58]. Even though IACT limits are weaker than those obtained from the Fermi-LAT satellite measurements in the GeV mass range [59], they complement the latter in the $\mathrm{TeV}$ mass range.

\subsection{Observation of the galactic center and halo}

The Galactic Center (GC) is a prime target for DM searches with IACTs. In 2004, H.E.S.S. published the observation of the GC and its interpretation in terms of DM during an observation campaign of about $50 \mathrm{~h}$ [20]. The resulting gamma-ray spectrum, obtained from a 40 standard deviations significant signal, was consistent with a power law and no significant features as expected at e.g. the DM mass cutoff — were detected. The analysis was performed first considering that the entire gammaray flux was coming from dark matter annihilation (instead of the plausible standard astrophysical sources like the central $\mathrm{BH}$ or closeby SNR). This provides lower limits on the possible DM mass scale at more than $10 \mathrm{TeV}$. In a second analysis, by considering a superposition of a power-law and different possible DM spectra, the results excluded compatibility with any DM model. Later observations from H.E.S.S. of the galactic center were published in Refs. [60, 61, 62], specially focused on a better localization of the gamma-ray emission, while no additional DM interpretation was given. Still, questions about the origin of the gamma rays and the emission mechanism remain open. In short, no conclusive evidence from DM emission could be drawn from the MW center because of the strong contribution from background emission of other nature.

For Milky-Way sized DM halo, the radial DM density profiles is obtained from N-body simulations (e.g. Aquarius [1], Via Lactea II [2]) and from observations. The former can be described by Einasto and NFW parameterizations. Large differences between both parameterizations occur if they are extrapolated down to the very center of the halo, where the NFW profile is more strongly peaked. However, at distances larger than $10 \mathrm{pc}$, the difference is reduced to a factor of two. This idea was used by H.E.S.S. which searched for the DM signal in a region with a projected galactocentric distance of $45-150$ $\mathrm{pc}$ that corresponds to an angular distance of $0.3-1.0^{\circ}$. In this ring, in addition, the contamination from gamma-ray sources is naturally avoided. The results were presented in Ref. [21] 
and resulted in the most constraining upper limits on DM annihilations from IACTs, as shown in Fig. 2. We remark that those limits are valid for cuspy profiles like the NFW and the Einasto ones. However, experimental observations tends to predict more shallow profile toward the galactic center (so-called "cored" profiles, see, e.g., [63]). All in all, the DM modelization of the galactic center region remains a complex task, because of the expected strong interplay with the baryons and the stellar winds and shocks, still to be clarified (see e.g. discussions in $[64,65,66])$.

Also MAGIC and VERITAS have observed the Galactic Center in these years, confirming the results from H.E.S.S. [67, 68]. However, this target is best observable from the Southern Hemisphere, where H.E.S.S. is located. At the location of MAGIC and VERITAS, the GC culminates low in the horizon, which results on one hand in a significant higher energy threshold for observation, but on the other hand in a considerably higher sensitivity at high energies than in vertical observation. Both instruments are still conducting campaigns on this target, and publications are expected in the coming years.

\subsection{Constraints for line emission}

Gamma-ray line signatures can be expected due to selfannihilation or decay of DM particles in space. Such a signal would be readily distinguishable from astrophysical gamma-ray sources that in most cases produce continuous spectra. There have been recent claims of hint of a line-like signal at about $130 \mathrm{GeV}$ in the Fermi data of the Galactic Center region [69, 70] which has received a huge attention. If confirmed, the WIMP particle should have a mass of about $m_{\chi} \sim 130 \mathrm{GeV}$ and annihilation rate (assuming Einasto profile) of $\langle\sigma v\rangle_{\gamma \gamma}=$ $1.27 \times 10^{-27} \mathrm{~cm}^{3} \mathrm{~s}^{-1}[2]$. Using data collected with H.E.S.S., upper limits on line-like emission were obtained in the energy range between $500 \mathrm{GeV}$ and $25 \mathrm{TeV}$ for the central part of the Milky Way halo and for extragalactic observations, complementing limits obtained with the Fermi-LAT instrument at lower energies [71]. No statistically significant signal could be found. For monochromatic gamma-ray line emission, flux limits were obtained for the central part of the Milky Way halo and extragalactic observations, respectively. For a DM particle mass of $1 \mathrm{TeV}$, limits on the velocity-averaged DM annihilation cross section $\langle\sigma v\rangle$ reach the level of $10^{-27} \mathrm{~cm}^{3} \mathrm{~s}^{-1}$, based on the Einasto parameterization of the Galactic DM halo density profile. Additional limits are calculated in [15] using the $158 \mathrm{~h}$ observation on Segue 1. In general, they are more than one order of magnitude worse than those from H.E.S.S. but possibly more solid, because in DSGs a core or a cusp profile results in J-factors that differ by a factor of few $[72,4,73]$.

\subsection{Constraints for all-electrons searches}

Among the cosmic rays impinging the Earth, a small but important fraction is constituted by electron and positrons (collectively called "all-electrons" in this context). When impinging the atmosphere, the initiate electromagnetic showers, which are indistinguishable from those initiated by gamma-rays, for what matters IACTs.
In the last few years, the all-electrons spectrum has shown unexpected behavior energies at energy larger than $100 \mathrm{GeV}$. The ATIC balloon claimed the observation of a bump at above $300 \mathrm{GeV}$ [74], which was partially disproved by the very precise measurements obtained with the Fermi-LAT satellite [75]. Still, in both cases, a discrepancy between the theoretical predictions and the observational results were extremely significant. In the same year, H.E.S.S. developed a specific analysis for electrons, based on the selection of "electron-like" events in regions far from gamma-ray sources and subtraction of the remaining hadronic cosmic ray background using simulations, and could measure with high precision the spectrum from $300 \mathrm{GeV}$ to $1.2 \mathrm{TeV}$ using $230 \mathrm{~h}$ of observation of the extragalactic dark sky [27, 28]. Later, MAGIC confirmed these results but with less significance and smaller energy coverage [29]. More recently, the AMS-02 satellite provided more precise data (see link [76] that includes data from the other experiments mentioned here). The anomalies in the $e^{-}$and $e^{+}$fluxes are generally interpreted as a new component with a harder spectrum and a higher $e^{+}$ratio than what is expected from secondary electrons induced by the galactic CR propagation in the galaxy. Many scenarios involving DM (annihilation/decay), nearby pulsars or inhomogeneity in the galactic CRs have been proposed to interpret the data [see, e.g., 77, 78].

The results obtained with H.E.S.S. and MAGIC are extremely important in the overall scenario. First of all, they provide an independent measurement, with different systematics compared to other experiments. This is specially important because the different results are sometimes not compatible within the systematics, which could shadow experimental or intrinsic not yet understood uncertainties. Secondly, the IACTs data could extend to energies larger than $1 \mathrm{TeV}$, where AMS-02 and FermiLAT are at least less sensitive. This region is extremely important for the DM searches, in case of DM mass at the TeV, because it is where a spectral cutoff is expected. More results are expected on this kind of measurements in the following years.

\subsection{Constraints on antiparticles separately}

In addition to what described in the previous section, in principle, IACTs can also observe separately electrons and positrons (as well as proton / antiproton or any charged particle / antiparticle) by using the fact that if the telescope is observing at a certain distance close enough to the Moon, the Moon itself blocks some cosmic rays creating a hole in the flux which is shifted along an axis perpendicular to the geomagnetic field and verse depending on the charge of the particle. The amplitude and direction of the deviation depend on the energy and charge of the cosmic ray. For example, at $500 \mathrm{GeV}$, the electron or positron shadow is shifted $3-4^{\circ}$ away from the Moon. This technique is currently under investigation by the MAGIC [30] and VERITAS experiment. However, no results have been published yet, and the feasibility of the technique has still to be proved.

The shape of the $e^{+}$ratio above $100 \mathrm{GeV}$, particularly at the $300-800 \mathrm{GeV}$ range, is crucial to discriminate between these models and to understand the origin of the electron spectrum anomalies. PAMELA, Fermi-LAT and AMS-02 among others, 
measured the $e^{+}$ratio up to about $350 \mathrm{GeV}$ [79]. Again, groundbased telescopes have in principle sensitivities larger than satellite for positrons above $350 \mathrm{GeV}$. Currently, the positron spectrum has very smooth features from 10 to $350 \mathrm{GeV}$, and therefore, the observation of some spectral features above this range would constitute an extremely important probe for possible DM interpretations.

\section{The future with the Cherenkov Telescope Array}

The Cherenkov Telescope Array (CTA) (see Ref. [80] for a full description and Ref. [81] for a shorter version also presented at this conference series) is a project for a next-generation observatory for very high energy $(\mathrm{GeV}-\mathrm{TeV})$ ground-based gammaray astronomy, currently in its design phase, and foreseen to be operative a few years from now. Several tens of telescopes of 3-4 different sizes, distributed over a large area, will allow for a sensitivity about a factor 10 better than current instruments such as H.E.S.S, MAGIC and VERITAS, an energy coverage from a few tens of $\mathrm{GeV}$ to several tens of $\mathrm{TeV}$, and a field of view of up to $10 \mathrm{deg}$. The search for new physics beyond the Standard Model (SM) of particle physics is among the key science drivers of CTA [82].

CTA is expected to improve the prospects for DM searches with IACTs on the following basis: $a$ ) naively, the improved sensitivity, compared to current instruments, will generically improve the probability of detection, or even identification of DM, through the observation of spectral features, $b$ ) the energy range will be extended. At low energies, this will allow overlap with the Fermi-LAT or other similar future instruments, and will provide sensitivity to WIMPs with low masses. For WIMPs with mass larger than about $100 \mathrm{GeV}$, CTA will be the experiment with highest sensitivity; $c$ ) the increased FOV (about $10 \mathrm{deg}$ versus $2-5 \mathrm{deg}$ ) with a much more homogeneous sensitivity, as well as the improved angular resolution, will allow for much more efficient searches for extended sources like galaxy clusters and spatial anisotropies; $d$ ) finally, the improved energy resolution will allow much better sensitivity to the possible spectral feature in the DM-generated photon spectrum. The observation of a few identical such spectra from different sources will allow both precision determination of the mass of the WIMP and its annihilation cross-section.

In Ref. [54], a detailed report of the performance for CTA DM searches, estimated employing Monte Carlo simulations of different possible CTA realizations, is given, as well as for other fundamental physics issues, like the possible existence of axion-like particles, expected violation of Lorentz Invariance by quantum gravity effects. In [54, Fig. 23], the expected performance of CTA for WIMPs annihilating purely into $b \bar{b}$ in $100 \mathrm{~h}$ observation at DSGs, galaxy clusters, and at the Galactic Center Halo is shown, together with extrapolation of the FermiLAT performance for 10 years of data. As expected, the best results are achieved for the observation in the vicinity of the Galactic Center, where the thermal annihilation cross-section for WIMP DM of $10^{-26} \mathrm{~cm}^{3} \mathrm{~s}^{-1}$ is at reach. This would be the first time that ground-based Cherenkov telescopes could reach this sensitivity level.

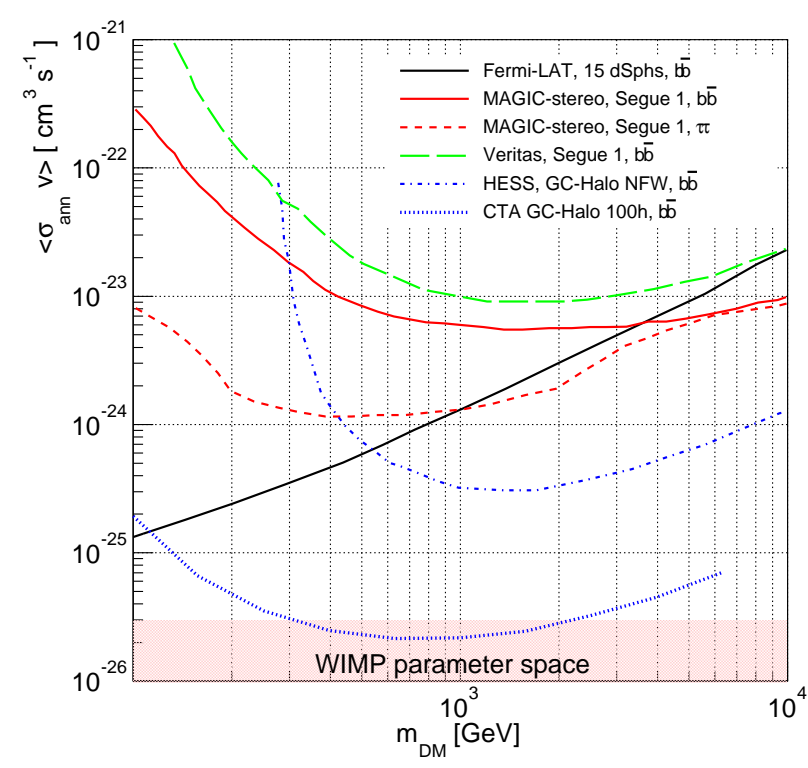

Figure 2: Comparison of some exclusion lines for the Fermi-LAT observation of 15 combined DSGs for $b \bar{b}$ (solid black) [85], H.E.S.S. observation of the galactic center halo for the NFW (dot-dashed blue [21]) for the $b \bar{b}$ channel, MAGIC-stereo observations of the Segue 1 DSG for the $b \bar{b}$ (solid red) and $\tau^{+} \tau^{-}$(dashed red) channels [15], Veritas observations of the Segue 1 DSG for $b \bar{b}$ (dashed green) [14], and for the estimation for $100 \mathrm{~h}$ observation at the galactic center halo with CTA (thick dashed blue) [54]. More details in the text.

It is worth noting here that on July 2012 the H.E.S.S. collaboration inaugurated the fifth telescope, a $28 \mathrm{~m}$ diameter dish telescope which is currently the largest Cherenkov dish ever built [83]. This will result in a larger sensitivity below 100 $\mathrm{GeV}$ with interesting expectations specially for the $130 \mathrm{GeV}$ line searches [84].

\section{Conclusions}

In Fig. 2 we collect few results on the exclusion curves for WIMP annihilation cross-section. On the bottom left side of the plot, we see the exclusion power of Fermi-LAT observation of 15 combined DSGs for the $b \bar{b}$ (solid black line) [85] We also show the best limit obtained on DSG observation with Segue 1 observed during $158 \mathrm{~h}$ with the MAGIC stereo experiment again for the $b \bar{b}$ (solid red line) and $\tau^{+} \tau^{-}$(dashed red line) channels [15]. These two channels somehow represent two extreme cases, a very soft spectrum $(b \bar{b})$ and a very hard spectrum $\left(\tau^{+} \tau^{-}\right)$. One can see that because of the better sensitivity of MAGIC at higher energies, the harder $\tau^{+} \tau^{-}$channel is better constrained. The same target was observed in $48 \mathrm{~h}$ of observation with Veritas (dashed green) [14]. In blue, we show the H.E.S.S. exclusion curve from the galactic center halo for the NFW (dot-dashed blue line) [21] for the $b \bar{b}$ channel only. Finally, we show estimates for $100 \mathrm{~h}$ observation of the Galactic Center halo region with CTA (thick dashed blue, [54]) considering again a NFW profile.

The detection of gamma-rays provides complementary information to other experimental probes of particle DM, especially that of direct detection, because CTA could be able to 
access a fraction of the parameter space not accessible otherwise [86]. With respect to particle searches at the LHC, the comparison is not straightforward, as LHC results are usually strongly related to specific models, and general conclusions are somewhat model dependent. In any case, LHC discovery of $\mathrm{DM}$, would prompt the need for proof that the particle is actually consistent with the astrophysical DM. A concrete scenario has been analyzed [87] in the case of a SUSY model in the so-called co-annihilation region. Simulated LHC data were used to derive constraints on the particle physics nature of the $\mathrm{DM}$, with the result that the LHC alone is not able to reconstruct the neutralino composition. The situation improves if the information from a detection of gamma-rays after the observation of the Draco DSG by IACT like CTA is added to the game: the internal degeneracies of the SUSY parameter space are broken and including IACT results allows us to fully interpret the particle detected at the LHC as the cosmological DM. In the other case where the LHC will not detect any physics beyond the Standard Model, predictions were made in the context of the CMSSM [88] indicating that the mass of the neutralino will be bound to be close to the $\mathrm{TeV}$ scale. In this scenario, MAGIC, H.E.S.S. and VERITAS and, even more, CTA could be the only instrument to be able to detect and identify a WIMP candidate with masses beyond some hundreds GeV. Finally, we mention that CTA will open a new possibility in detecting DM with IACTs based on the detection of spatial anisotropies in the diffuse extragalactic gamma-ray sky [89, 90].

Acknowledgment: I gratefully acknowledge J. Conrad, C. Farnier, R. Ong, M. Fornasa and A. Smith for comments on the manuscript as well as the MAGIC and CTA collaborations. This work is funded by University of Padova.

\section{References}

[1] J. L. Feng, Naturalness and the Status of Supersymmetry, 2013. UCI-TR2013-01.

[2] T. Bringmann, C. Weniger, Gamma Ray Signals from Dark Matter: Concepts, Status and Prospects, Phys.Dark Univ. 1 (2012) 194-217.

[3] J. L. Feng, Dark Matter Candidates from Particle Physics and Methods of Detection, Ann.Rev.Astron.Astrophys. 48 (2010) 495.

[4] A. Charbonnier, et al., Dark matter profiles and annihilation in dwarf spheroidal galaxies: prospectives for present and future $\gamma$-ray observatories - I. The classical dwarf spheroidal galaxies, MNRAS 418 (2011) 1526-1556.

[5] M. Wood, et al., A Search for Dark Matter Annihilation with the Whipple 10m Telescope, ApJ 678 (2008) 594.

[6] A. Abramowski, et al. (HESS Coll.), HESS observations of the globular clusters NGC 6388 and M 15 and search for a Dark Matter signal, ApJ 735 (2011) 12.

[7] J. Albert, et al. (MAGIC Coll.), Upper limit for gamma-ray emission above $140-\mathrm{GeV}$ from the dwarf spheroidal galaxy Draco, ApJ 679 (2008) $428-431$.

[8] V. Acciari, et al. (VERITAS Coll.), VERITAS Search for VHE Gammaray Emission from Dwarf Spheroidal Galaxies, ApJ 720 (2010) 11741180 .

[9] F. Aharonian, et al. (HESS Coll.), Observations of the Sagittarius Dwarf galaxy by the HESS experiment and search for a Dark Matter signal, AP 29 (2008) 55-62.

[10] F. Aharonian, et al. (HESS Coll.), A search for a dark matter annihilation signal towards the Canis Major overdensity with H.E.S.S, ApJ 691 (2009) $175-181$.

[11] E. Aliu, et al. (MAGIC Coll.), Upper limits on the VHE gamma-ray emission from the Willman 1 satellite galaxy with the MAGIC Telescope, ApJ 697 (2009) 1299-1304.
[12] A. Abramowski, et al. (HESS Coll.), HESS constraints on Dark Matter annihilations towards the Sculptor and Carina Dwarf Galaxies, AP 34 (2011) 608-616.

[13] J. Aleksic, et al. (MAGIC Coll.), Searches for Dark Matter annihilation signatures in the Segue 1 satellite galaxy with the MAGIC-I telescope, JCAP 1106 (2011) 035.

[14] E. Aliu, et al. (VERITAS Coll.), VERITAS Deep Observations of the Dwarf Spheroidal Galaxy Segue 1, PR D85 (2012) 062001.

[15] J. Aleksi, S. Ansoldi, L. Antonelli, P. Antoranz, A. Babic, et al., Optimized dark matter searches in deep observations of Segue 1 with MAGIC, JCAP 1402 (2014) 008.

[16] J. S. Perkins, et al. (Veritas Coll.), TeV gamma-ray observations of the perseus and abell 2029 galaxy clusters, ApJ 644 (2006) 148-154.

[17] J. Aleksic, et al. (MAGIC Coll.), MAGIC Gamma-Ray Telescope Observation of the Perseus Cluster of Galaxies: Implications for Cosmic Rays, Dark Matter and NGC 1275, ApJ 710 (2010) 634-647.

[18] A. Abramowski, et al. (HESS Coll.), Search for Dark Matter Annihilation Signals from the Fornax Galaxy Cluster with HESS, ApJ 750 (2012) 123.

[19] T. Arlen, et al. (VERITAS Coll.), Constraints on Cosmic Rays, Magnetic Fields, and Dark Matter from Gamma-Ray Observations of the Coma Cluster of Galaxies with VERITAS and Fermi, ApJ 757 (2012) 123.

[20] F. Aharonian, et al. (HESS Coll.), HESS observations of the Galactic Center region and their possible dark matter interpretation, PRL 97 (2006) 221102.

[21] A. Abramowski, et al. (HESS Coll.), Search for a Dark Matter annihilation signal from the Galactic Center halo with HESS, PRL 106 (2011) 161301.

[22] F. Aharonian, et al. (HESS Coll.), Search for Gamma-rays from Dark Matter annihilations around Intermediate Mass Black Holes with the HESS experiment, PR D78 (2008) 072008.

[23] M. Doro, et al., Dark Matter searches at intermediate mass black hole with the MAGIC telescope, in: Proc. of the 30th ICRC, Merída, Mexico, 2007.

[24] A. Abramowski, et al. (HESS Coll.), Search for photon line-like signatures from Dark Matter annihilations with HESS, PRL 110 (2013) 041301.

[25] D. Nieto, et al. (MAGIC Coll.), The search for galactic dark matter clump candidates with Fermi and MAGIC, in: Procs, of the 31st ICRC, 2011.

[26] A. Geringer-Sameth (VERITAS), The VERITAS Dark Matter Program, in: Procs. 4th Fermi Symp., 2013.

[27] F. Aharonian, et al. (HESS Coll.), Probing the ATIC peak in the cosmicray electron spectrum with H.E.S.S, AA 508 (2009) 561.

[28] F. Aharonian, et al. (HESS Coll.), The energy spectrum of cosmic-ray electrons at TeV energies, PRL 101 (2008) 261104.

[29] D. Borla Tridon, et al. (MAGIC Coll.), Measurement of the cosmic electron plus positron spectrum with the MAGIC telescopes, in: Proc. of the 32th ICRC, 2011.

[30] P. Colin, et al. (MAGIC Coll.), Probing the CR positron/electron ratio at few hundreds $\mathrm{GeV}$ through Moon shadow observation with the MAGIC telescopes, in: Proc. of the 32th ICRC, 2011.

[31] L. E. Strigari, et al., A common mass scale for satellite galaxies of the Milky Way, Nature 454 (2008) 1096-1097.

[32] J. Diemand, et al., Clumps and streams in the local dark matter distribution, Nature 454 (2008) 735-738.

[33] V. Springel, et al., The Aquarius Project: the subhaloes of galactic haloes, MNRAS 391 (2008) 1685-1711.

[34] L. E. Strigari, et al., Redefining the Missing Satellites Problem, ApJ 669 (2007) 676-683.

[35] M. Boylan-Kolchin, et al., Too big to fail? The puzzling darkness of massive Milky Way subhaloes, Mon.Not.Roy.Astron.Soc. 415 (2011) L40.

[36] L. E. Strigari, Galactic Searches for Dark Matter, 2012. Submitted to Phys.Rev.

[37] R. Essig, et al., Indirect Dark Matter Detection Limits from the UltraFaint Milky Way Satellite Segue 1, PR D82 (2010) 123503.

[38] G. Lamanna, et al. (H.E.S.S. Coll.), Sagittarius dwarf spheroidal galaxy observed by H.E.S.S, 2013. Proceedings of the 33rd International Cosmic Ray Conference (ICRC2013), Rio de Janeiro (Brazil).

[39] L. Bergstrom, D. Hooper, Dark matter and gamma-rays from Draco: MAGIC, GLAST and CACTUS, PR D73 (2006) 063510.

[40] J. Aleksic, J. Rico, M. Martinez, Optimized analysis method for indirect dark matter searches with Imaging Air Cherenkov Telescopes, JCAP 
$1210(2012) 032$.

[41] B. Zitzer (VERITAS), Dark Matter Annihilation Limits from Dwarf Galaxies using VERITAS, 2013. ArXiv e-Print:1307.8367.

[42] L. Pieri, et al., Dark Matter Annihilation in Substructures Revised, MNRAS 384 (2008) 1627.

[43] R. C. Hartman, et al. (EGRET), The Third EGRET catalog of high-energy gamma-ray sources, ApJS 123 (1999) 79.

[44] P. Nolan, et al. (Fermi-LAT Coll.), Fermi Large Area Telescope Second Source Catalog, ApJS 199 (2012) 31.

[45] D. Nieto, et al., A search for possible dark matter subhalos as IACT targets in the First Fermi-LAT Source Catalog, in: Procs, of the 3rd Fermi Symposium, 2011.

[46] J. F. Navarro, et al., A Universal density profile from hierarchical clustering, ApJ 490 (1997) 493-508.

[47] G. Bertone, et al., A new signature of dark matter annihilations: Gammarays from intermediate-mass black holes, PR D72 (2005) 103517.

[48] G. Voit, Tracing cosmic evolution with clusters of galaxies, Rev.Mod.Phys. 77 (2005) 207-258.

[49] A. Pinzke, et al., Prospects of detecting gamma-ray emission from galaxy clusters: cosmic rays and dark matter annihilations, Phys. Rev. D 84 (2011) 123509.

[50] M.-A. Sanchez-Conde, et al., Dark matter searches with Cherenkov telescopes: nearby dwarf galaxies or local galaxy clusters?, JCAP 1112 (2011) 011.

[51] L. Gao, et al., Where will supersymmetric dark matter first be seen?, MNRAS 419 (2012) 1721.

[52] J. Aleksíc, et al., Detection of very high energy gamma ray emission from NGC 1275 by the MAGIC telescopes, AA 539 (2011).

[53] J. Aleksic, et al. (MAGIC), Detection of very high energy gamma-ray emission from the Perseus cluster head-tail galaxy IC 310 by the MAGIC telescopes, 2010. ArXiv e-print:1009.2155.

[54] M. Doro, et al. (CTA Coll.), Dark Matter and Fundamental Physics with the Cherenkov Telescope Array, AP 43 (2013) 189-214.

[55] J. Aleksic, et al. (MAGIC Coll.), Constraining Cosmic Rays and Magnetic Fields in the Perseus Galaxy Cluster with $\mathrm{TeV}$ observations by the MAGIC telescopes, A\&A 541 (2012) A99.

[56] F. Aharonian (HESS Coll.), Very high energy gamma-ray observations of the galaxy clusters Abell 496 and Abell 85 with H.E.S.S, AA 495 (2008) 27.

[57] F. Aharonian, et al., Constraints on the multi-TeV particle population in the Coma galaxy cluster with HESS observations, AAP 502 (2009) $437-443$.

[58] A. Acciari, et al. (VERITAS Coll.), VERITAS Upper Limit on the VHE Emission from the Radio Galaxy NGC 1275, ApJ 706 (2009) L275L280.

[59] A. Abdo, et al., Observations of Milky Way Dwarf Spheroidal galaxies with the Fermi-LAT detector and constraints on Dark Matter models, ApJ 712 (2010) 147-158.

[60] F. Aharonian, et al. (HESS Coll.), Simultaneous HESS and Chandra observations of Sagittarius A* during an X-ray flare, AA 492 (2008) L25.

[61] F. Aharonian, et al. (HESS Coll.), Spectrum and variability of the Galactic Center VHE gamma-ray source HESS J1745-290, AA 503 (2009) 817.

[62] F. Acero, et al. (HESS Coll.), Localising the VHE gamma-ray source at the Galactic Centre, MNRAS 402 (2010) 1877-1882.

[63] F. Donato, et al., A constant dark matter halo surface density in galaxies, MNRAS 397 (2009) 1169-1176.

[64] G. A. Gomez-Vargas, et al., Constraints on WIMP Annihilation for Contracted Dark Matter in the Inner Galaxy with the Fermi-LAT JCAP10 (2013) 029.

[65] G. Ogiya, et al., The connection between the cusp-to-core transformation and observational universalities of DM halos, 2013. ArXiv ePrint:1309.1646.

[66] A. Di Cintio, et al., The dependence of dark matter profiles on the stellar to halo mass ratio: a prediction for cusps vs cores, 2013. ArXiv ePrint:1306.0898.

[67] J. Albert, et al. (MAGIC Coll.), Observation of gamma-rays from the galactic center with the magic telescope, ApJ 638 (2006) L101-L104.

[68] M. Beilicke (VERITAS Coll.), The Galactic Center Region Imaged by VERITAS from 2010-2012, AIP Conf.Proc. 1505 (2012) 462-465.

[69] T. Bringmann, et al., Fermi lat search for internal bremsstrahlung signatures from dark matter annihilation, JCAP 07 (2012). 054.
[70] C. Weniger, A tentative gamma-ray line from dark matter annihilation at the fermi large area telescope, JCAP 08 (2012). 007.

[71] A. Abdo, et al., Fermi LAT Search for Photon Lines from 30 to $200 \mathrm{GeV}$ and Dark Matter Implications, PRL 104 (2010) 091302.

[72] T. Bringmann, M. Doro, M. Fornasa, Dark Matter signals from Draco and Willman 1: Prospects for MAGIC II and CTA, JCAP 0901 (2009) 016.

[73] G. D. Martinez, A Robust Determination of Milky Way Satellite Properties using Hierarchical Mass Modeling, 2013. ArXiv e-Print:1309.2641.

[74] J. Chang, et al., An excess of cosmic ray electrons at energies of 300-800 $\mathrm{GeV}$, Nature 456 (2008) 362-365.

[75] A. A. Abdo, et al. (Fermi-LAT), Measurement of the Cosmic Ray e+ plus e- spectrum from $20 \mathrm{GeV}$ to $1 \mathrm{TeV}$ with the Fermi Large Area Telescope, PRL 102 (2009) 181101.

[76] http://www.ams02.org/2013/07/new-ams-data5-all-electron-spectru 2013.

[77] I. Cholis, et al., The Case for a 700+ GeV WIMP: Cosmic Ray Spectra from ATIC and PAMELA, Phys.Rev. D80 (2009) 123518.

[78] S. Profumo, Dissecting cosmic-ray electron-positron data with Occam's Razor: the role of known Pulsars, Central Eur.J.Phys. 10 (2011) 1-31.

[79] M. Aguilar, et al. (AMS-02 Coll.), PRL 110 (2013) 141102.

[80] M. Actis, et al. (CTA Consortium), Design concepts for the Cherenkov Telescope Array CTA: An advanced facility for ground-based highenergy gamma-ray astronomy, Exper.Astron. 32 (2011) 193-316.

[81] M. Doro (CTA Coll.), CTA - A Project for a New Generation of Cherenkov Telescopes, NIM A630 (2011) 285-290.

[82] J. Hinton, et al. (CTA Coll.), A new era in gamma-ray astronomy with the cherenkov telescope array, AP 43 (2013) 1-356.

[83] B. Giebels (H.E.S.S. Coll.), Status and recent results from H.E.S.S, 2013. 4th Fermi Symp., Monterey, USA.

[84] L. Bergstrom, et al., Investigating Gamma-Ray Lines from Dark Matter with Future Observatories, JCAP 1211 (2012) 025.

[85] M. Ackermann, et al. (Fermi-LAT Collaboration), Dark Matter Constraints from Observations of 25 Milky Way Satellite Galaxies with the Fermi Large Area Telescope, Phys.Rev. D89 (2014) 042001.

[86] L. Bergstrom, T. Bringmann, J. Edsjo, Complementarity of direct dark matter detection and indirect detection through gamma-rays, Phys.Rev. D83 (2011) 045024.

[87] G. Bertone, et al., Complementarity of Indirect and Accelerator Dark Matter Searches, Phys.Rev. D85 (2012) 055014.

[88] G. Bertone, et al., Dark Matter Searches: The Nightmare Scenario, JCAP 1201 (2012) 004.

[89] M. Fornasa, J. Zavala, M. A. Sanchez-Conde, J. M. Siegal-Gaskins, T. Delahaye, et al., Characterization of Dark-Matter-induced anisotropies in the diffuse gamma-ray background, MNRAS, 429, 1529 (2013).

[90] J. Ripken, A. Cuoco, H.-S. Zechlin, J. Conrad, D. Horns, The sensitivity of Cherenkov telescopes to dark matter and astrophysically induced anisotropies in the diffuse gamma-ray background, JCAP 01 (2014) 049 (2012). 